\title{
NiTiCu shape memory alloy: Superplastic elongation during thermal cycling
}

\author{
P. Bassani and S. Besseghini \\ CNR-TeMPE, Corso Promessi Sposi 29, 23900 Lecco, Italy
}

\begin{abstract}
We report on experimental results obtained in the characterization of a $\mathrm{Ni}_{45} \mathrm{Ti}_{50} \mathrm{Cu}_{5}$ shape memory alloy tested in order to verify its properties at the light of the use in actuators. In this field long term stability of the recovery properties and low hysteresis values are the main requests. Tests were performed in an "ad-hoc" developed device with a maximum number of cycles equal to 5000 and an applied load of 180MPa. Various thermal treatments and cold-working conditions were tested on several specimens in order to identify the combinations that can ensure the best functional properties at the light of the use in actuators. Calorimetric measurements were systematically performed on specimen taken at the beginning and at the end of the cycling procedure. Results confirm that low temperatures and short time thermal treatments account for higher stability in the cycling properties. Surprisingly it has been noted that specimens submitted to complete anneal thermal treatment, in spite of a large plastic deformation during thermomechanical cycling, exhibit only small changes in the recovery properties. A NiTiCu full annealed wire cycled up to rupture can account for a stored plastic deformation up to $60 \%$ of the initial length, at the same time demonstrating a complete shape memory effect of $5 \%$. A detailed investigation of the material submitted to this treatment was done in order to clarify its behavior.
\end{abstract}

\section{INTRODUCTION}

NiTi alloys with copper substituting a certain amount of $\mathrm{Ni}$ are interesting alternative of the binary alloys for actuator applications. Interest for ternary alloys relays mainly on the following arguments: reduction of transformation temperature sensitiveness to chemical composition; reduction of thermal hysteresis; reduction of mechanical hysteresis and better cycling behavior [1]. The possible use of these alloys as actuator materials is strictly connected to their fatigue life. As for the other shape memory alloys, fatigue life is influenced by the boundary conditions (e.g. test temperatures, maximum strain allowed) and test method, as clearly pointed out by several papers related to cycling properties of binary NiTi alloys (2). The transformations involved are important too. Ternary NiTiCu alloys in isothermal conditions (temperature constant, variable load) received some investigation. More attention was devoted to non isothermal test (constant load, variable temperature) but generally limited to few thermal cycles, only occasionally up to 10000 cycles (3). In the present paper results about the effect of thermal cycling on the behavior of a $\mathrm{Ni}_{45} \mathrm{Ti}_{50} \mathrm{Cu}_{5}$ alloy are presented. The $\mathrm{Ni}_{45} \mathrm{Ti}_{50} \mathrm{Cu}_{5}$ alloy exhibits a single thermoelastic martensitic transformation whose product phase is similar to the one present in the binary NiTi alloy. The $B C C$ austenite (B2) transforms on cooling to a distorted monoclinic phase (B19') $(4,5)$.

In this paper we focus our attention on the behavior of fully annealed specimens. Of course this thermal treatment cannot be considered optimal from the point of view of cyclic stability but, during testing, the treated specimens shown specific effect worth of a deeper investigation. A roughly constant recoverable strain was detected up to rupture in spite of a severe plastic elongation of the specimen. We will refer to this phenomenon as a kind of superplastic deformation or creep deformation during cycling. 


\section{EXPERIMENTAL}

The material used in this study was a NiTiCu alloy with $5 \%$ at of Copper substituting an even percentage of $\mathrm{Ni}$. The alloy was produced in and induction furnace by meiting pure metals in a graphite crucible under vacuum followed by casting in a water cooled copper mould. The ingots were subsequently Plasma remelted, canned in steel and hot extruded to a $\phi=16 \mathrm{~mm}$ bar. A standard deformation process by means of hot working, cold working with intermediate anneals and cold drawing with intermediate anneal transformed the extruded bars to wires of $0.75 \mathrm{~mm}$ in diameter. The residual cold work at the end of the deformation process was roughly of $30 \%$.

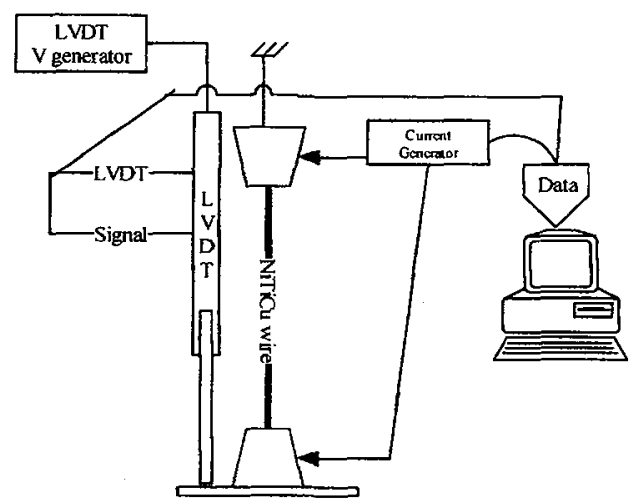

Figure 1: schematic layout of the cycling device used to test long term actuation capabilities of NiTiCu Specimens for testing in an "ad-hoc" developed apparatus shape memory alloys were cut (200 to $300 \mathrm{~mm}$ in length) and heat treated at $450^{\circ} \mathrm{C}(1.8 \mathrm{Ks}$, WQ and $3.6 \mathrm{Ks}$, WQ) in air, and at $750^{\circ} \mathrm{C}$ for $3.6 \mathrm{Ks}$ in an evacuated quartz tube followed by water quench (WQ). Specimens submitted to the last procedure are here considered as "full annealed" specimens.

Specimens for differential scanning calorimetry (DSC), for each thermal treatment, were cut from the wires before and after cycling procedure and lightly polished with emery papers and chemical cleaning.

The DSC measurements were performed with a Seiko DSC $220 \mathrm{C}$, with a scanning rate of $5^{\circ} \mathrm{C} / \mathrm{min}$, in the temperature range $\left[-50^{\circ} \mathrm{C} ; 100^{\circ} \mathrm{C}\right]$. Thermomechanical cycling test were performed by applying a static load (stress value equal to $180 \mathrm{MPa}$ ) to the NiTiCu wire. No strain limiter was applied. The wire was electrically actuated; the current level is chosen in order to heat up the wire in few seconds. During cycling the only value detected were maximum and minimum strain corresponding to the maximum elongation at the end of the cooling step and maximum recovery at the end of the heating step. On occurrence of a predefined number of actuation cycles a slower cycle was performed with acquisition of the whole strain-temperature curve both on heating and on cooling. The whole set of tests was performed with a computer controlled setup with data acquisition capability. Tests were performed at room temperature and with a maximum number of cycles equal to 5000 . A schematic view of the cycling device is reported in Figure 1

\section{RESULTS}

Before and after the cycling procedure, a DSC test has been performed on each wire, to evaluate the transformation temperatures and the modification induced by the cycling itself on the transformation.

Figure 2(a) shows a comparison of the thermograms measured on samples treated at $450^{\circ} \mathrm{C}$ and at $750^{\circ} \mathrm{C}$ for different times. As expected, the treatment at $750^{\circ} \mathrm{C}$ rises the transformation temperatures. Furthermore the shape itself of the calorimetric peak is changed. In the fully annealed specimen the increased asymmetry of the peak suggests the lock-in of a multistep transformation. On cooling, just below Ms, there is evidence of a small peak merged with the main one. Specimens treated at lower temperatures show a dependence from the treatment times with a slight decrease of the transformation temperatures for increasing times.

The results presented in Figure 2(b) compares the calorimetric curves obtained on specimens taken from the cycled wires. The specimens were taken from the central part of the wires. Thermal cycling greatly affected the DSC results; in all cases except the $\mathrm{Ni}_{45} \mathrm{Ti}_{50} \mathrm{Cu}_{5}$ wire treated at $750^{\circ}$ for one hour (that will be discussed later), there are common features. The peaks have a lower spread on the temperature scale, there's a slight increase in transformation temperatures whilst the transformation hentalpy is not greatly affected. The heating branch of the thermograms, i.e. the ones related to the reverse transformation, show the greatest modifications. In the case of the specimen treated at $450^{\circ} \mathrm{C}$ for $60^{\prime}$ and cycled 5000 times the peak becomes even split in two. 
Let's turn to the full annealed specimen $\left(750^{\circ} \mathrm{C} 1 \mathrm{~h}\right)$ which shows a different behavior. The transformation peak shifts to lower temperatures and the transformation hentalpy reduces. It has to be noted that the total number of cycles is much lower in this case as the wire broke after only 600 cycles. For comparison in Figure 2(b) the calorimetric curve before the cycling procedure is reported too.

(a)

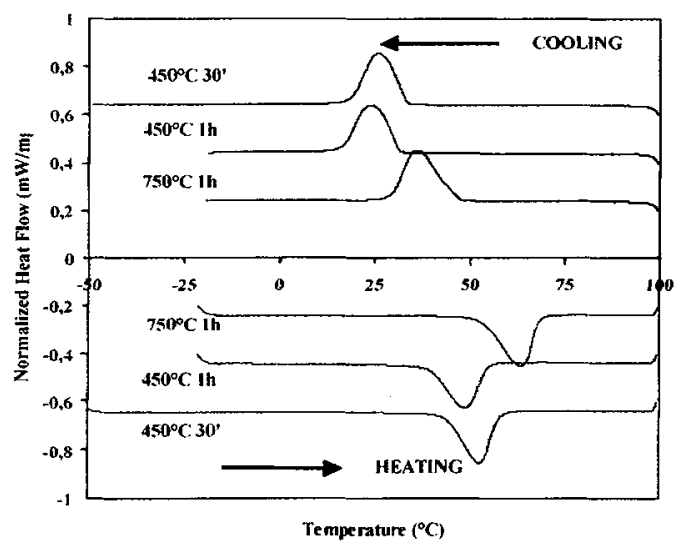

(b)

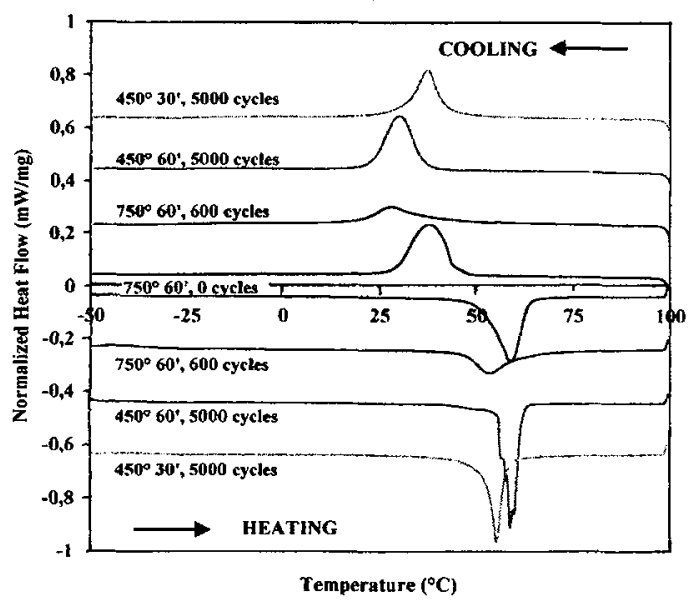

Figure 2 Comparison of the calorimetric curves obtained. (a) samples before the cycling procedure. (b) specimens taken from the cycled wires at the end of the cycling procedure or on failure.

From this very preliminary observations, and taking care of the great difference in the number of cycles performed, a simple conclusion can be drawn. The specimens treated at lower temperatures still retain some degree of cold-working and the main effect of cycling is to rearrange and accommodate the defect structure so that the transformation is trained to take place with the aid of the internal stresses. As a consequence transformation peaks becomes tighter and shift slightly to higher temperatures. Otherwise the full annealed specimen is submitted to a kind of work hardening in which new defects and structures are created by the cycling procedure itself. This can explain the progressive reduction of the hentalpy of transformation and the great similarity of the behavior with specimens submitted to low degree of coldworking.

In the following Figure 3 the results obtained during the cycling procedure are plotted as a function of the number of total actuation cycles. The Stress Assisted Two Way Shape Memory Effect (SATWSME) is plotted as a function of the number of cycles. It is expressed both as actual elongation in $\mathrm{mm}$ and as actual strain in percent. The strain is evaluated as $\left(\mathrm{L}_{\max }-\mathrm{L}_{\min }\right) / \mathrm{L}_{\max }{ }^{*} 100$ where $\mathrm{L}_{\max }$ is the elongation at the beginning of each heating run and $L_{\min }$ is the length of the wire at the end of the heating ramp corresponding to the maximum contraction of the wire. In such a way the strain is always evaluated as an instant value related to the actual length of the wire at the beginning of the cycle. The total elongation of the wire is computed as $\left(\mathrm{L}_{\max }-\mathrm{L}_{0}\right) / \mathrm{L}_{0} * 100$ where $\mathrm{L}_{0}$ is the length of the wire at the beginning of the test and $\mathrm{L}_{\max }$ the same as above. The results presented in Figure 3 refer to two different specimens, the first one is the wire treated at $750^{\circ} \mathrm{C}$ for $\mathrm{lh}$ that is a full anneal thermal treatment. There is evidence of a giant elongation of the wire during thermal cycling with a final plastic deformation very close to $90 \%$. The total elongation shows three regions. In the initial stages the wire quickly elongates of about $10 \%$ with a roughly exponential trend. Afterwards instead of the usual stabilization of the properties it continues to elongate with a linear trend (roughly $10 \%$ each 100 cycles) up to about 450 cycles at which a substantial increase of the rate of elongation sets in and brings the wire to failure after only 600 cycles. For comparison in Figure 4(b) the same results for a specimen treated at $450^{\circ} \mathrm{C}$ for $30^{\prime}$ is reported. This treatment correspond to the one that exhibited the best stabilization of the recovery properties. To allow direct comparison of the two results the two graphs have been reported on the same scale. It is clear the 
huge deformation suffered by the annealed wire. At the same time the recoverable strain in both the specimens is surprising similar and about $5 \%$.

In order to gain a deeper understanding of the modification induced by the cycling procedure in the fully annealed specimen metallographic observations have been performed. Metallographic investigation both with optical microscopy (MO) and scanning electron microscopy (SEM) didn't demonstrate any specific mesoscopic arrangement of the martensitic structure. The cycled specimen featured a very fine dispersion of microcracks on the surface and in the interior of the specimen. Figure 4 exemplifies these cases: here a comparison between a non cycled (a) and a cycled wire (b) is presented.

The cycled wires clearly present the fine dispersion of microcracks on the surface.

(a)
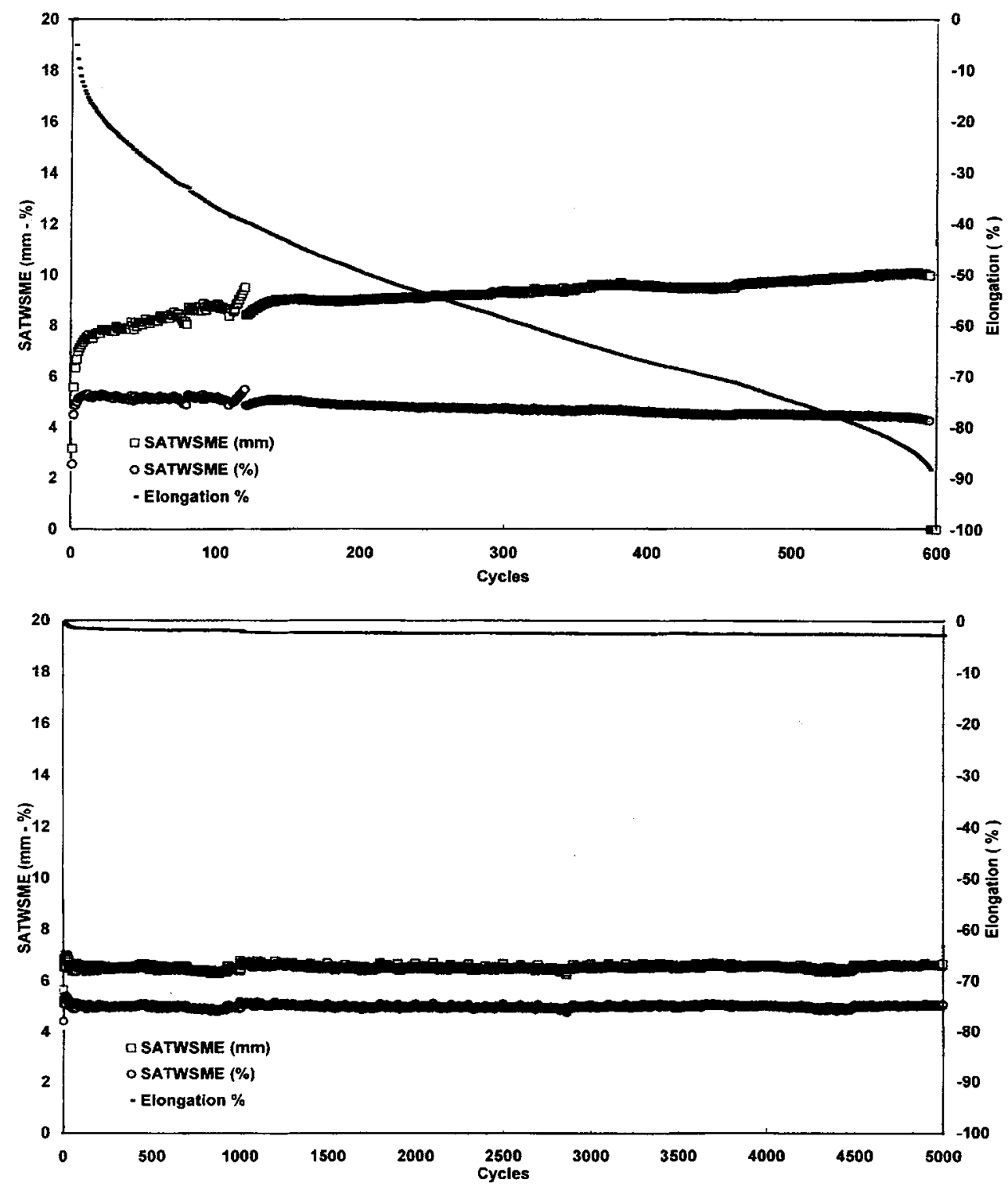

Figure 3 (a) Cycling behavior of the full annealed specimen $\left(750^{\circ} \mathrm{C} / 60^{\prime}\right)$ (b) Cycling behavior of the $450^{\circ} \mathrm{C} / 30^{\prime}$ annealed wire. 
(a)

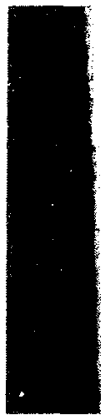

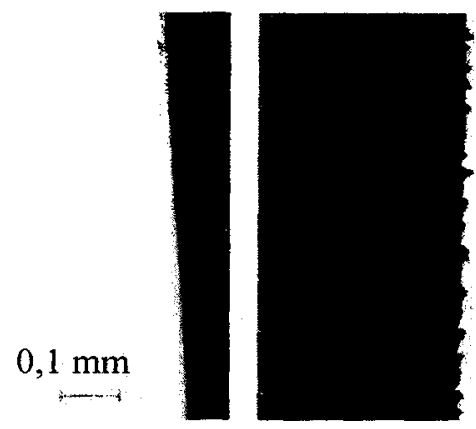

(b)

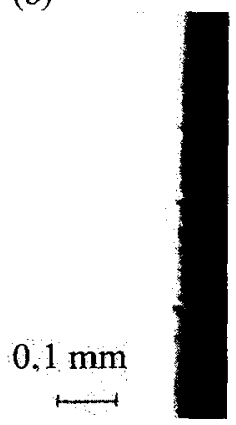

Figure 4: metallography of the sample treated at $750^{\circ} \mathrm{C} / 60^{\prime}$ before of the cycling procedure and after 600 thermomechanical cycles.

\section{DISCUSSION}

It is quite difficult to understand how a full annealed wire of NiTiCu can maintain the capability of recovering up to $5 \%$ once it has elongated more than $50 \%$ over its initial length. It should be noted that the total deformation suffered by the NiTiCu wire before fracture was near $90 \%$. This value compares with a 40-50\% maximum elongation values observed during preliminary tensile tests and routine plastic deformation operations. Furthermore when the wire during standard plastic deformation procedure reaches its upper work hardening limit, the fracture is very brittle.

However it is well known that increasing cold working amount in NiTiCu brings to a decrease of the calorimetric signal and that $30 \%$ cold worked wires give no evidence of martensitic transformation [6]. On the contrary (see Figure 2(b)) the calorimetric signal is present. For comparison with similar results it can be argued that the calorimetric signal is similar to the one of a slightly cold worked material, say not more than $10 \%$.

It is concluded that the giant deformation exhibited by the full annealed wire didn't correspond to a real plastic straining of the wire. Likely different mechanisms sum up giving rise to the macroscopi deformation of the wire. It has to be noted that tests performed simply hanging the dead load to the wire in its martensitic state didn't show any elongation over time. As a consequence the transformation is a necessary condition to activate the mechanisms responsible of the elongation. The interaction between the austenite/martensite interfaces and a progressive accommodation of slipped martensite is probably the key mechanism.

The three stages behavior of the total elongation curve of the fully annealed specimen (Figure 3-b) can be explained at the light of the metallographic results. At the beginning the usual process of dislocations build up and assessment of the transformational paths takes places. As a consequence we observe the rapid increase of the total length ad usual stabilization effects. Afterwards the material enters the zone in which the stored defects in non annealed materials are able to stabilize transformation. This is not the case for the annealed wire. Progressively adjacent parts of the wire suffer plastic deformation promoting elongation of the wire. The remaining part continue to guarantee the total recovery properties of the system. Finally it can be argued that at about 450 cycles the formation of cracks at the surface takes place or their density reach some kind of critical value that has the effect to reduce the effective area of the wire. The actual stress increases quickly leading to failure the wire. 


\section{Acknowledgments}

Thanks are due to Mr. M. Pini for specimens preparation and precious help in experimental work.

\section{References}

1 T.H. Nam, T. Saburi, K. Shimizu, Mat. Trans., JIM, Vol.31,. No. 11 (1990)

2 H. Tobushi, T. Hasimoto, Y. Shimeno, K. Tarata, Mat. Scie. Forum, Vol 327-328(2000),pp 151-154

3 R. Zadno, J.W. Simpson, M.A. Imran, Proc. of the Forst Int. Conf. On Shape Memory and Superelastic Technologies, Asilomar Conf. Center, Pacific Grove, California, USA, (1994)

4 T. Saburi, Proc. Int. Conf. On Martensitic Transformation (1992), Monterey, California, USA

5 Tae Hyun Nam, Toshio Saburi, Yoshiyuki nakata, Ken'ichi Shimizu, Mat. Trans., JIM, Vol. 31 , No. $12(1990)$, pp 1050-1056

6 S. Besseghini, L. Mirri, A. Tuissi, 7th International Conf. On Adaptive Structures, sept. 23-25, 1996, Roma, p. 277 